\title{
Design and Performance Evaluation of Priority Vehicle for Emergency Services in VANET
}

\author{
Saket Tiwari ${ }^{1}$, Vipin Bondre ${ }^{2}$ \\ PG Scholar, Electronics and Telecommunication Engineering, YCCE, Nagpur, India ${ }^{1}$ \\ Professor, Electronics and Telecommunication Engineering, YCCE, Nagpur, India ${ }^{2}$
}

\begin{abstract}
Today's era is the era of fastest speed considering from the evolution of wheel. In the process of development, there has been a drastic increase in the speed as well as the number of vehicles. With the increase in number of vehicles there the aroused the need for Intelligent Transport Scenario with efficiency in terms of reduced travel time, zero congestion (traffic jams), etc. Thus the way for using intelligent traffic system got fulfilled in the form Vehicular Ad hoc Network (VANET). The use of VANET include the communication between the vehicular nodes to circulate information like speed, destination of a node, proposed way, etc. to other nodes. In all the applications related to Vehicular Ad Hoc Network include traffic management, traffic bypassing, etc. The other cited application of VANET that is coming into light is in Emergency services. In addition to the use of siren as in ambulance, fire trucks, police cars, etc. there can be use of communication between the emergency vehicle and other vehicles so that other vehicles can be warned about presence of emergency vehicle. In this paper, the VANET scenario has been simulated wherein the emergency vehicle communicates with other vehicles. The simulation has been carried out using MOVE, Simulation of Urban Mobility (SUMO) and Network Simulator (NS2).
\end{abstract}

Keywords: Priority Vehicle, VANET, AOMDV, SUMO, MOVE, NS2.

\section{INTRODUCTION}

The improved lifestyle has increased the number of vehicles running on road using limited resources which has consequently increased the amount of congestion Along with the increased need of transportation and the intelligent transport, the incorporation of wireless communication has become the prime need. Also the safety institutions are increasingly depending on the incorporated wireless communication techniques during the emergency operations like fire rescue, road accident, etc. During the emergency response trip, the emergency vehicle itself is at highest risk of getting into an accident as it is travelling at much higher speed than other vehicles. Thus the incorporation of communication units in the emergency vehicle will be of higher utility in order to safeguard the vehicle.

The above mentioned requirement can be solely accomplished with the presence of VANET in the scenario. VANETs are termed to be an important development that can provide significant contribution in emergency services and road safety. VANET is the major sub part of Mobile Ad Hoc Network. In all the VANET network incorporate methodology of communication between the vehicular nodes in order to transmit the vehicular data of one node to other nodes which can be used for adding intelligence to the traffic flow and increase the efficiency of transportation.

In this paper, the major objective is to reduce the travel time of emergency vehicle and to avoid the congestion in its path by informing the presence of emergency vehicle before handed to other normal vehicles. Every driver must be familiar with the situation when the sound of siren from approaching emergency vehicle is heard and struggle for finding the correct move is started. If the destination and the path that will be followed by emergency vehicle, is known by the other vehicles, then the reaction and movements of other vehicles will be in controlled and efficient way. In addition with the problem in regards of delay, another seriously heading issue is the crashes of the emergency vehicle itself. The main requirement for communication in emergency vehicle during emergency situation is real time communication and low delay transmission. Thus the above mentioned requirements have been evaluated with the evaluation of parameters like throughput, end to end delay and packet delivery ratio. The parameters have been evaluated against varying number of regular vehicles in the scenario.

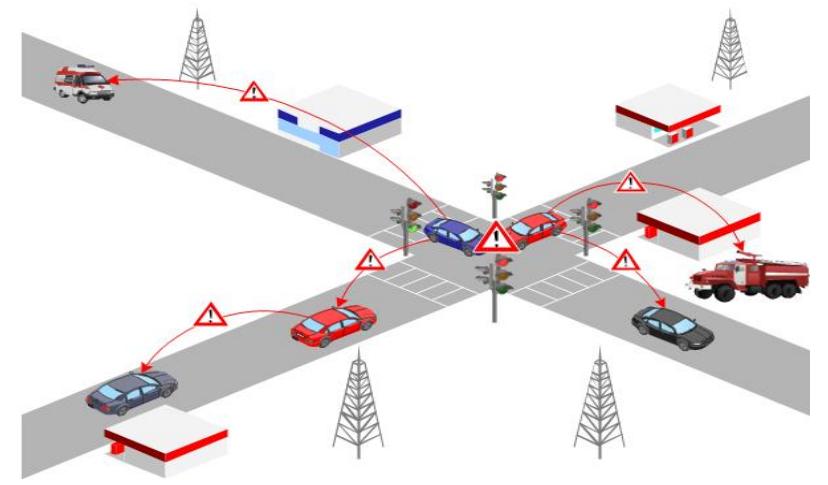

Fig. 1.Vehicular traffic using VANET 
The routing protocol used for evaluation is of reactive category i.e. Ad Hoc on Demand Multipath Distance Vector routing protocol which calculates multiple paths to destination which is very useful in the highly dynamic VANET network in case of path failure.

Figure 1 shows the example of Vehicular Ad Hoc Network (VANET) scenario which shows the transmission of information between the vehicular nodes. The emergency vehicle is proposed such that it transmits its destination and other information to all the other vehicles in the scenario.

\section{OBJECTIVE}

The major objective of the work presented in this paper is to design and simulate a VANET scenario containing the normal vehicles and the priority vehicle (Emergency vehicle).The designed scenario will be used for simulating data transmission i.e. the packet transmission from the priority node to regular nodes.

In all the major task involved after designing the scenario is to evaluate the network performance by studying packet parameters, like throughput, end to end delay and packet delivery ratio. The parameters will be studied under varying number of regular vehicles in the scenario.

The simulation of the described situation is performed with the help of three interfaces sequentially. The softwares used are Simulation of Urban MObility (SUMO 0.12.3) and Network Simulator (NS 2.34) along with MOVE (MObility model generator for VEhicular networks) to generate supporting files for SUMO.

\section{III.METHODOLOGY}

The following section elaborates the procedure incorporated while execution of work. The procedure involves developing the required topology first, getting the mobility pattern of nodes over the designed topology and performing network simulation over the designed topology. The protocol used for evaluation is AOMDV (Ad hoc On-demand Multipath Distance Vector) which is an ad hoc routing protocol which provides multiple paths between source and destination nodes which are useful in case of path failure.

\section{A. Topology}

The topology creation includes designing the scenario map using MOVE. The process includes making nodes i.e. traffic signals and road intersections. Then the roads are selected between the nodes which are called as edges. Then the .net file is obtained from .nod.xml and .edg.xml files. Furthermore the vehicle movement is needed which is createdto get .rou.xml file. The vehicles can be created automatically by defining vehicle flow and turn ratio or manually by fixing vehicle maximum speed, vehicle route, maximum length of vehicle, etc. the .cfg file and .rou files are combined to get sumo configuration file which is used for simulating vehicle movement on SUMO.
The user interface of MOVE is shown in Figure 2 which include options like node select, edge select, map create, vehicle movement creation, etc.

After getting support files for SUMO simulation using MOVE, the simulation is visualised using SUMO-GUI.

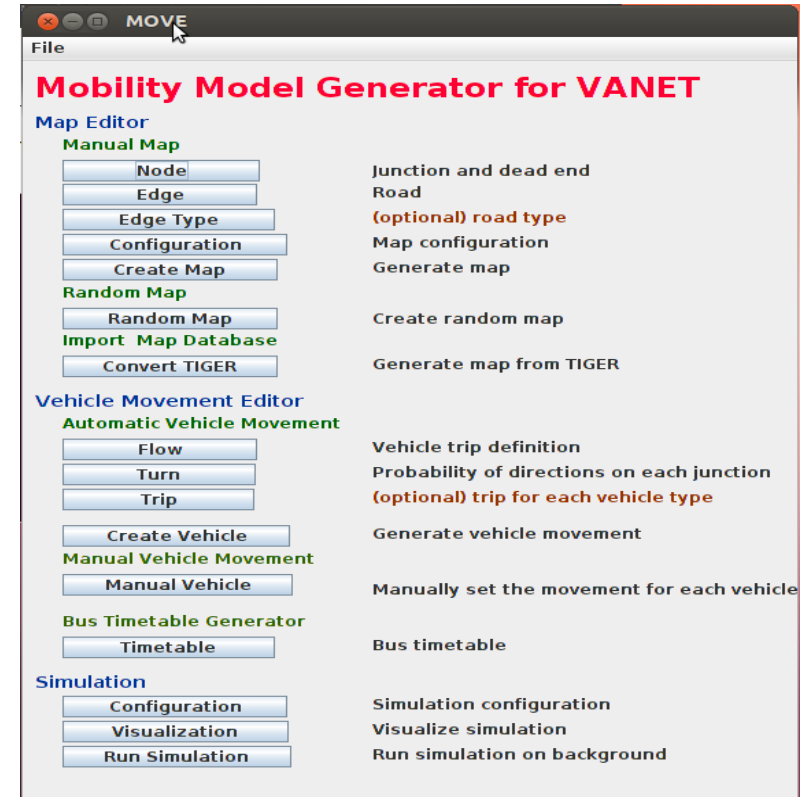

Fig. 2. Modelling of topology in MOVE

Sumo is an open source traffic simulator which provides mobility of vehicular nodes simulated in it, which is used for performing network simulation in NS2. The SUMO version used in this paper is 0.12 .3 as MOVE is supported only by SUMO 0.12.3. The SUMO configuration (.cfg) file is required for running simulation in SUMO. The configuration file is obtained from the combining of node file, edge file and the route file.

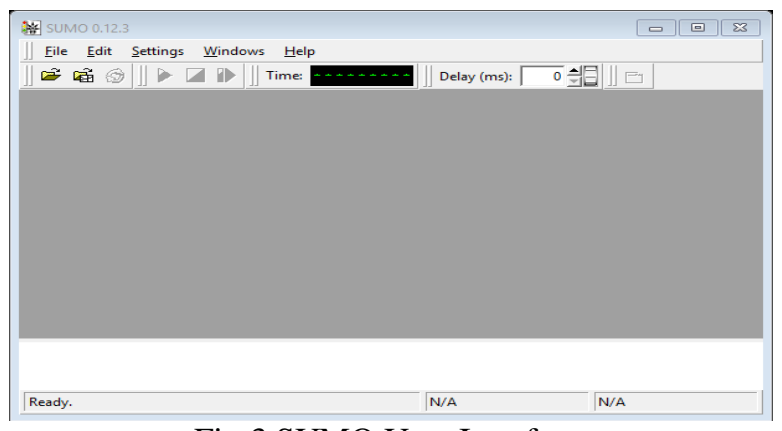

Fig.3 SUMO User Interface

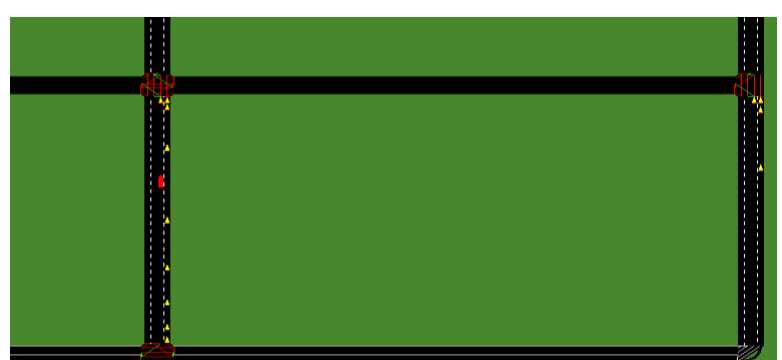

Fig. 4. Visualization of road traffic in SUMO 
Figure 3 shows the user interface of SUMO 0.12.3. The instance of simulation is shown in Figure 4.

\section{B. Routing protocol}

The multipath, reactive routing protocol AOMDV is employed in this work. AOMDV is the Ad Hoc routing protocol which finds multiple paths between the source to destination. These multiple paths are brought into operation when the active path between the nodes fail.

Basically, routing protocol in ad hoc networks play an important role while packet dissemination from one node to another. The end point simulation in this work is done in NS2 which operates and simulate at packet level. Thus the routing protocol employed will decide how the packets are transmitted, the path for the flow of packets, whether the packets will be transmitted in single hop or multiple hops.

IEEE 802.11 WLAN standard is used while simulating the vehicular network in this work and the routing protocol used is AOMDV. Analysis in [1] and [2] shows improved performance when AOMDV is employed in the wireless scenario.

\section{Traffic Connection}

The User Datagram Protocol (UDP) with Constant Bit Rate (CBR) is employed for generation of traffic in NS2. Here CBR is a Quality of Service (QoS) parameter under use. The advantage of using UDP is there is no connection establishment involved before transmission starts unlike in TCP. Hence the time required for packet transmission in UDP is less as compared with TCP.

\section{SIMULATION}

The simulation of the work is carried out in three phases in step.
1) MOVE
2) $\quad \operatorname{SUMO}(0.12 .3)$
3) NS2 (2.34)

\section{A. Stage-I}

The stage one include designing of scenario map. The scenario map has been designed on SUMO. The map is designed in the form of .xml files in SUMO. The xml files for creating map and vehicles in SUMO are created with the help of MOVE as shown in Figure2. Firstly the roads are designed by first creating nodes and then the edges connecting nodes. The node file and edge file are combined to get the net file (map file). Then the running vehicles are created. The vehicles can be created manually as well as automatically.

Here manual vehicle creation is done by setting maximum vehicle speed, vehicle length, number of rotes to be followed and number of vehicles following each specified route, etc. The vehicle file is obtained as .rou.xml file. This route file and net file are combined to get the sumo configuration file which is run in the SUMO-GUI. After running the SUMO configuration file, sumo trace file is obtained. This sumo trace file (.sumo.tr) is useful for getting the movement of the vehicular nodes while simulation in NS2.

\section{B. Stage-II}

The output of the SUMO simulation is the sumo trace file which is used along with the net file for obtaining the TCL script to be run over in NS2. The simulation is done by varying the number of nodes as 10,20, 30 and 40. The routing protocol used herein is AOMDV which is a multipath protocol. For all the simulations node $(0)$ is the emergency node. Thus a single emergency node is considered along with varying number of regular vehicles while simulation.

1) Simulation Parameters

TABLE I. PARAMETERS USED FOR SIMULATION

\begin{tabular}{|c|c|}
\hline Parameter & Used values \\
\hline Channel & Wireless \\
\hline Radio propagation & Two ray ground \\
\hline Antenna type & Omni-directional \\
\hline Link layer type & LL \\
\hline Interface Queue type & Queue/Drop Tail/PriQueue \\
\hline Max packet in queue & 50 \\
\hline MAC type & 802.11 \\
\hline Routing protocol & AOMDV \\
\hline Traffic types & UDP/CBR \\
\hline Number of nodes & $10,20,30,40$ \\
\hline Packet size & 500 \\
\hline
\end{tabular}

\section{RESULTS}

TABLE II. NETWORK VALUES EVALUATED

\begin{tabular}{|l|l|l|l|l|}
\hline & $\begin{array}{l}\mathbf{1 0} \\
\text { Nodes }\end{array}$ & $\begin{array}{l}\mathbf{2 0} \\
\text { Nodes }\end{array}$ & $\begin{array}{l}\text { 30 } \\
\text { Nodes }\end{array}$ & $\begin{array}{l}\text { 40 } \\
\text { Nodes }\end{array}$ \\
\hline $\begin{array}{l}\text { End to End } \\
\text { Delay }\end{array}$ & $0.44 \mathrm{mS}$ & $0.37 \mathrm{mS}$ & $0.31 \mathrm{mS}$ & $\begin{array}{l}0.28 \\
\mathrm{mS}\end{array}$ \\
\hline PDR & $94.29 \%$ & $93.06 \%$ & $92.83 \%$ & $91.96 \%$ \\
\hline Throughput & 90.8 & $\begin{array}{l}133.64 \\
\mathrm{kBps}\end{array}$ & $\begin{array}{l}153.86 \\
\mathrm{kBps}\end{array}$ & $\begin{array}{l}17.38 \\
\mathrm{kBps}\end{array}$ \\
\hline
\end{tabular}

The parameters like End to End delay, PDR and throughput are evaluated by transmitting packets using UDP/CBR connection.

\section{PERFORMANCE EVALUATION}

Figure 5 shows the instance of simulation in NS2 Network Animator. The simulation has been done in NS2.34. The routing protocol that has been evaluated for priority vehicles is AOMDV. The UDP/CBR has been employed for traffic generation. The simulation-evaluation of parameters has been performed under varying regular vehicles and single priority vehicle. 


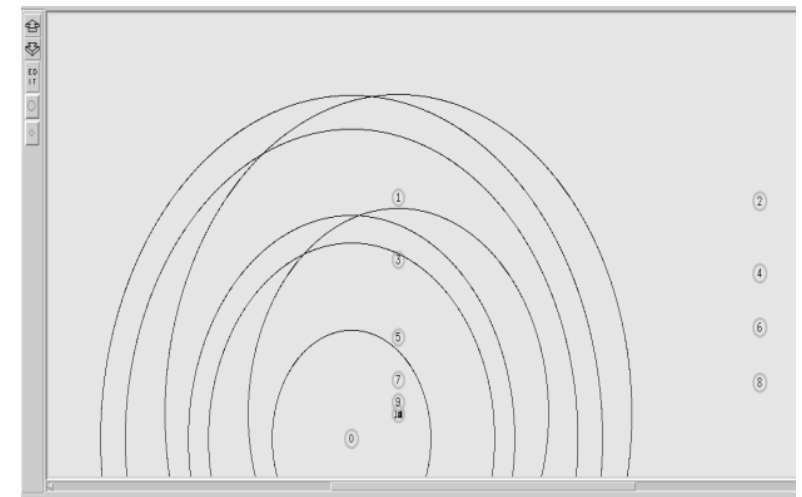

Fig. 5. Instance of simulation in NS2

Figure 6 shows the plot of average end to end delay vs. number of nodes. The value has been decreasing with increasing number of nodes and saturating as the number of nodes increase.

Here the use of UDP in place of TCP helps decreasing the delay as UDP does not include connection establishment before transmission. The parameter of delay is of importance here as the work has been carried out on priority vehicle in scenario.

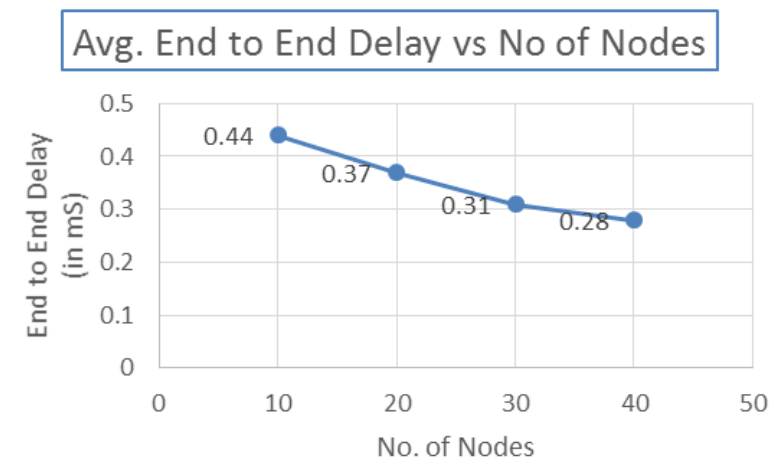

Fig. 6. Avg. E2E Delay Vs. No of nodes

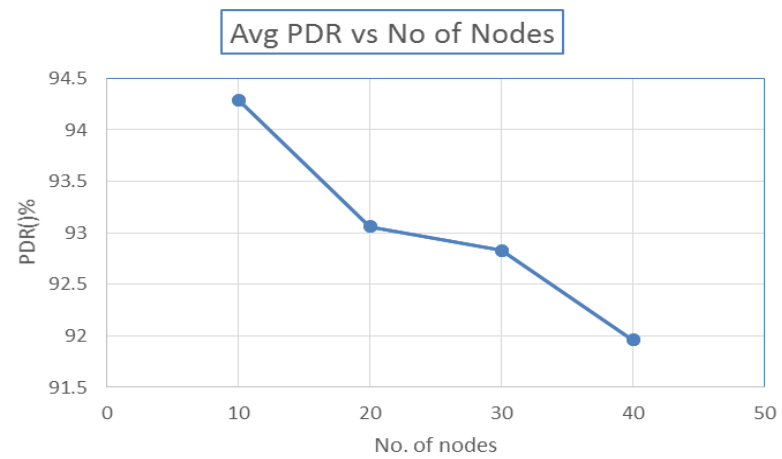

Fig.7. Avg. PDR Vs. No of nodes

Figure 7 shows the plot of average PDR vs. number of nodes. The values are in the range above $90 \%$.

Figure 8 shows the graph of average throughput vs. number of nodes which shows improvement in throughput with increase in number of nodes. Here the decrement in PDR has been observed due to usage of UDP.

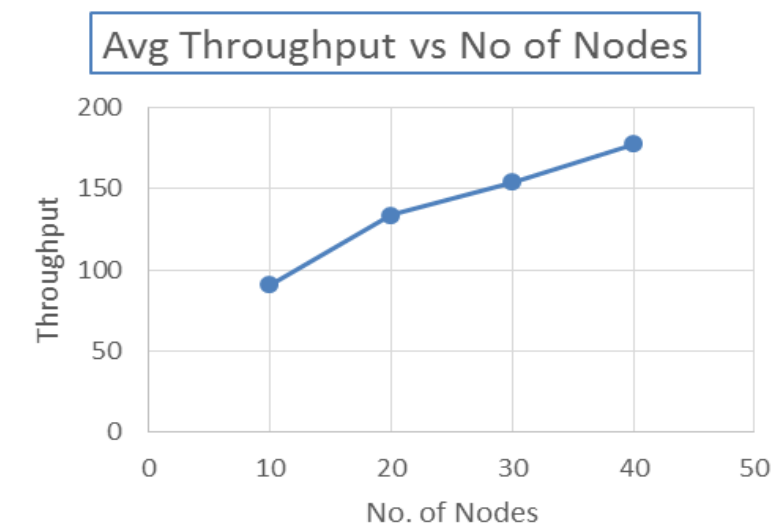

Fig. 8. Avg. Throughput Vs. No of nodes

\section{CONCLUSION}

The priority vehicles running on the roads can create huge loss during the operation, as the regular may get caught in its way. Thus, the use of wireless communication to inform the presence of priority vehicle and the destination of the same to regular vehicle will be of great help. The multipath routing protocol AOMDV has also been evaluated here with satisfactory parameter values. It is to be noticed that by enabling and introducing VANET networking in priority vehicles will initially lead to the introduction of VANET in the market.

\section{REFERENCES}

[1] Dharmendra Sutariya and Shrikant Pradhan, "Evaluation of Routing Protocols for Vanets in City Scenarios," Emerging Trends in Networks and Computer Communications (ETNCC), 2011.

[2] Biao Han, Jie Li, Jinshu Su \&Jiannong Cao, "Self-Supported Cooperative Networking for Emergency Services in Multi-Hop Wireless Networks," IEEE Journal On Selected Areas In Communications, Vol. 30, No. 2, February 2012.

[3] Rostam Shirani, Faramarz Hendessi, Mohammad Ali Montazeri and Mohammad Sheikh Zefreh, "Absolute Priority for a Vehicle in VANET”, CSICC 2008, CCIS 6, pp. 955-959, 2008.@ SpringerVerlag Berlin Heidelberg 2008

[4] Mat'iasPighin, Pablo I. Fierens, "VANET for emergency vehicles: preliminary results", 2015 XVI Workshop on Information Processing and Control (RPIC) (C2015 IEEE.

[5] Soufiene Djahel, Mazeiar Salehie, Irina Tal and PooyanJamshidi, "Adaptive Traffic Management for Secure and Efficient Emergency Services in Smart Cities", Work in Progress session at PerCom 2013, San Diego.

[6] Yuma Moroi, KazumasaTakami, "A Method of Securing Priorityuse Routes for Emergency Vehicles Using Inter-vehicle and Vehicle-road Communication", 2015 7th International Conference on New Technologies, Mobility and Security (NTMS).

[7] M. Greis, Tutorial for the Network Simulator "NS" http://www.isi.edu/nsnam/ns/tutorial/index.html.

[8] D. M"uller, "Typische Gefahrenbei Einsatzfahrten des Rettungs dienstes," Institutf urVerkehrsrecht und Verkehrsverhalten Bautzen, vol. 37, 2007.

[9] Mei-Ju Su', Heng-Shuen C h e r $\sim$ G e ng,-Shiau Lin' ,Fong-Ming S h y ', Syi Su', Po-Hsun Cheng', Chung-Liang Shih', Jia-Wei Lin' ,Sao-Jie Chen', “Application of Wireless Network in a Medical Emergency Service Network,"7th International Workshop on Enterprise networking and Computing in Healthcare Industry, 2005.

[10] Andreas Buchenscheit, Florian Schaub, Frank Kargl, and Michael Weber, "A VANET-based Emergency Vehicle Warning System," Vehicular Networking Conference (VNC), 2009 IEEE. 
[11] Ricardo Macedo, Robson Melo, Aldri Santos, Michele Nogueira, "Experimental Performance Comparison of Single-path and Multipath Routing in VANETs,"Global Information Infrastructure and Networking Symposium (GIIS), 2014

\section{BIOGRAPHIES}

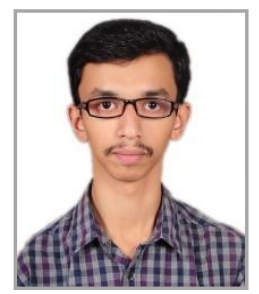

Saket Tiwari is currently pursuing his Master of Technology (M. Tech) program in Communication Engineering from Yeshwantrao Chavan College of Engineering, Nagpur, India. He has completed his Bachelor of Engineering (B.E) program in Electronics and Telecommunication Engineering from Nagpur University.

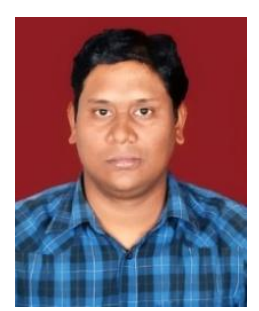

Mr. Vipin D. Bondre is currently working as Assistant Professor in Electronic and Telecommunication Department. He has done his B.E in Industrial Electronics from North Maharashtra University, Jalgaon and M. Tech from RTM, Nagpur University. Presently he is pursuing his Ph.D. from RTM Nagpur University in Wireless Communication. His area of Interest is Wireless Communication and Embedded Systems. He has published more than ten papers in international conference and journals and also working as a reviewer for many international conferences and Journals. He has about 10 years of experience in both industry and teaching. 